\title{
COMPUTABLE STRUCTURAL FORMULAS FOR THE DISTRIBUTION OF THE $\beta$-JACOBI EDGE EIGENVALUES
}

\author{
PETER J. FORRESTER AND SANTOSH KUMAR \\ To the memory of Richard Askey
}

\begin{abstract}
Авstract. The Jacobi ensemble is one of the classical ensembles of random matrix theory. Prominent in applications are properties of the eigenvalues at the spectrum edge, specifically the distribution of the largest (e.g. Roy's largest root test in multivariate statistics) and smallest (e.g. condition numbers of linear systems) eigenvalues. We identify three ranges of parameter values for which the gap probability determining these distributions is a finite sum with respect to particular bases, and moreover make use of a certain differential-difference system fundamental in the theory of the Selberg integral to provide a recursive scheme to compute the corresponding coefficients.
\end{abstract}

\section{o. Prologue}

The Selberg integral is the $N$-dimensional generalisation of the Euler $\beta$-integral

$$
J_{\lambda_{1}, \lambda_{2}, \beta, N}=\int_{0}^{1} d x_{1} \cdots \int_{0}^{1} d x_{N} \prod_{l=1}^{N} x_{l}^{\lambda_{1}}\left(1-x_{l}\right)^{\lambda_{2}} \prod_{1 \leq j<k \leq N}\left|x_{k}-x_{j}\right|^{\beta} .
$$

In a paper published in 1944 [45] Selberg derived the product of gamma function evaluation, specified by (1.2) below. As detailed in the review [27], knowledge of this result went almost completely unnoticed for over 30 years, until a number of coincidences led to the realisation that it provides a proof of the conjecture

(o.1)

$$
\frac{1}{(2 \pi)^{n / 2}} \int_{-\infty}^{\infty} \cdots \int_{-\infty}^{\infty} \prod_{j=1}^{n} e^{-t_{i}^{2} / 2} \prod_{1 \leq j<k \leq n}\left|t_{j}-t_{k}\right|^{2 \gamma} d t_{1} \cdots d t_{n}=\prod_{j=1}^{n} \frac{\Gamma(1+j \gamma)}{\Gamma(1+\gamma)}, \quad \operatorname{Re} \gamma>-1 / n,
$$

formulated by Mehta and Dyson [38] in the context of random matrix theory applied to nuclear physics.

Outside of the original paper, the now ex-conjecture (0.1) appeared in the first edition of Mehta's book [36] and also, in 1974, in the problem section of SIAM review [37]. From the source [7], we learn that it was from this latter publication that Askey took an active

Date: July 7, 2021. 
interest in this class of multiple integrals. At the beginning of the '8o's he was responsible for initiating the study of $q$-generalisations [6], and also supervised the well known $\mathrm{PhD}$ thesis of Morris [40] on the wider theory, by then understood to be related to root systems from the theory of Lie algebras.

In 1987 Aomoto's [3] work on proving (and extending) Selberg's result by deriving the recurrence in $\lambda_{1}$

$$
J_{\lambda_{1}+1, \lambda_{2}, \beta, N}=\prod_{p=0}^{N} \frac{\lambda_{1}+1+p \beta / 2}{\lambda_{1}+\lambda_{2}+2+(N-1+p) \beta / 2} J_{\lambda_{1}, \lambda_{2}, \beta, N}
$$

was published. This work, which was underpinned by a sophisticated viewpoint of the theory of multidimensional hypergeometric functions relating to de Rham cohomology, later summarised in the book [4], makes use of nothing beyond skilful application of integration by parts.

One of us (PJF) had the opportunity to visit Askey in Madison for two months from late August in 1988. In discussions, it came up how fundamental Askey viewed the recurrence relation viewpoint on the gamma function evaluation of the Euler integral - the case $N=1$ of (1.2), and how he often used it as extension material in a gifted high school student program as an example of powerful ideas which get missed in conventional undergraduate syllabi. However, as expressed in his 1975 book [5], Askey's broader program was "to study special functions not for their own sake, but to be able to use them to solve problems". In relation to the Selberg integral, a number of its uses and consequences to topics such as random matrix theory, conformal field theory, and statistical properties of the zeros of the Riemann zeta function are reviewed in [27]. Subsequently the Selberg integral has facilitated a number recent advances in the field of Gaussian multiplicative chaos [43].

In the present work we return to the recursive structures of the type introduced in [3], and contribute to Askey's broader program by identifying certain of their applications within random matrix theory.

\section{INTRODUCTION}

1.1. The Jacobi ensemble. In random matrix theory the Jacobi (or more precisely $\beta$-Jacobi) ensemble refers to the class of eigenvalue probability density functions

$$
\mathcal{P}^{\mathrm{J}}\left(x_{1}, \ldots, x_{N}\right):=\frac{1}{J_{\lambda_{1}, \lambda_{2}, \beta, N}} \prod_{l=1}^{N} x_{l}^{\lambda_{1}}\left(1-x_{l}\right)^{\lambda_{2}} \chi_{x_{l} \in(0,1)} \prod_{1 \leq j<k \leq N}\left|x_{k}-x_{j}\right|^{\beta} .
$$


Here $\chi_{J}=1$ for $J$ true, $\chi_{J}=0$ otherwise, and $J_{\lambda_{1}, \lambda_{2}, \beta, N}$ is the normalisation - it is the Selberg integral (see e.g. [19, Ch. 4]), given by

$$
J_{\lambda_{1}, \lambda_{2}, \beta, N}=\prod_{j=0}^{N-1} \frac{\Gamma\left(\lambda_{1}+1+j \beta / 2\right) \Gamma\left(\lambda_{2}+1+j \beta / 2\right) \Gamma(1+(j+1) \beta / 2)}{\Gamma\left(\lambda_{1}+\lambda_{2}+2+(N+j-1) \beta / 2\right) \Gamma(1+\beta / 2)} .
$$

An example of the Jacobi ensemble - although not then named as such — first revealed itself in studies of multivariate statistics; see e..g. [2, 41]. Specifically, let $X_{i}(i=1,2)$ be $n_{i} \times N\left(n_{i} \geq N\right)$ standard real Gaussian matrices, to be thought of as centred random data matrices. Let $W_{i}=X_{i}^{T} X_{i}(i=1,2)$ denote the corresponding $N \times N$ covariance matrices. One has that the eigenvalues of

$$
\left(\mathbb{I}+W_{1}^{-1} W_{2}\right)^{-1}
$$

form the Jacobi ensemble with parameters

$$
\left(\lambda_{1}, \lambda_{2}, \beta\right)=\left(\frac{1}{2}\left(n_{1}-N-1\right), \frac{1}{2}\left(n_{2}-N-1\right), 1\right) .
$$

This result can be generalised to the cases that the matrices $X_{i}$ contain standard complex Gaussian entries, or standard quaternion Gaussian entries, with the latter represented in $X_{i}$ as $2 \times 2$ complex blocks of the form

$$
\left[\begin{array}{cc}
z & w \\
-\bar{w} & z
\end{array}\right], \quad w, z \in \mathbb{C} .
$$

One has (see [19. Prop. 3.6.1]) that the eigenvalue probability density function of $(1.3)$ is now given the by the Jacobi ensemble with parameters

$$
\left(\lambda_{1}, \lambda_{2}, \beta\right)=\left(\frac{\beta}{2}\left(n_{1}-N+1-2 / \beta\right), \frac{\beta}{2}\left(n_{2}-N+1-2 / \beta\right), \beta\right),
$$

where $\beta=2$ (complex entries) and $\beta=4$ (quaternion entries). Note from (1.4) that setting $\beta=1$ in 1.5 specifies the case of real entries in 1.3 .

Square standard Gaussian random matrices $X$ can be used to construct Haar distributed unitary matrices according to

$$
S=X\left(X^{\dagger} X\right)^{-1 / 2}
$$

see e..g. [13]. Let $X$ (and thus $S$ ) be of size $L \times L$, and let $S_{n, N}(n \geq N)$ denote the top $n \times N$ sub-block of $S$ (since $S$ is Haar distributed, any $n$ rows and $N$ columns will do). Then, as a corollary of knowledge of the eigenvalues probability density function (1.3), it is possible 
to make use of [1.6 to show [11, 18] that for $L \geq n+N$ the square singular values of $S_{n, N}$ (i.e. the eigenvalues of $S_{n, N}^{+} S_{n, N}$ ) have density function given by the Jacobi ensemble with

$$
\left(\lambda_{1}, \lambda_{2}, \beta\right)=\left(\frac{\beta}{2}(n-N+1-2 / \beta), \frac{\beta}{2}(L-n-N+1-2 / \beta), \beta\right) .
$$

Again $\beta=1,2$ or 4 according to the entries of $X$ (and thus $S$ ) being real, complex or quaternion respectively.

Associated with the block decomposition of a real orthogonal matrix $S$ is the so-called cosine-sine (CS) expansion. Edelman and Sutton [16] showed that a sequence of Householder transformations can be applied to $S$ to obtain a real orthogonal matrix $S$ which is block bi-diagonal and has the same CS values. The top left diagonal matrix has the form

$$
\left[\begin{array}{cccc}
c_{N} & -s_{N} c_{N-1}^{\prime} & & \\
& c_{N-1} s_{N-1}^{\prime} & \ddots & \\
& & \ddots & -s_{2} c_{1}^{\prime} \\
& & & c_{1} s_{1}^{\prime}
\end{array}\right],
$$

where $c_{i}=\cos \theta_{i}, s_{i}=\sin \theta_{i}, c_{i}^{\prime}=\cos \phi_{i}, s_{i}^{\prime}=\sin \phi_{i}$, with all angles between o and $\pi / 2$. Moreover, with the notation $B[a, b]$ for the beta distribution, it is shown in [16] that

$$
\begin{array}{ll}
\cos ^{2} \theta_{j} \in \mathrm{B}[\beta(a+j) / 2, \beta(b+j) / 2] & (j=1, \ldots, N) \\
\cos ^{2} \phi_{j} \in \mathrm{B}[\beta j / 2, \beta(a+b+1+j) / 2] & (j=1, \ldots, N-1),
\end{array}
$$

and that the squared singular values of (1.7) have density given by (1.1) with

$$
\left(\lambda_{1}, \lambda_{2}, \beta\right)=\left(\frac{\beta}{2}(a+1)-1, \frac{\beta}{2}(b+1)-1, \beta\right)
$$

This construction thus realises the Jacobi ensemble for general parameters.

It is also true that for general parameters the probability density function (1), upon the change of variables $x_{l}=\sin ^{2} \phi_{l}$ is the ground state wave function for a quantum many body system on an interval of Calogero-Sutherland type [8].

1.2. Significance of the distribution of the largest or smallest eigenvalue. Denote by $E_{N}^{\mathrm{J}}\left(0 ;(s, 1) ; \lambda_{1}, \lambda_{2}, \beta\right)$ the probability that there are no eigenvalues in the interval $(s, 1)$ of the Jacobi ensemble 1 .I. . The density function $p_{\max }^{(N)}\left(s ; \lambda_{1}, \lambda_{2}, \beta\right)$ for the distribution of the largest eigenvalue is obtained from $E_{N}^{\mathrm{J}}$ according to

$$
p_{\max }^{(N)}\left(s ; \lambda_{1}, \lambda_{2}, \beta\right)=\frac{d}{d s} E_{N}^{\mathrm{J}}\left(0 ;(s, 1) ; \lambda_{1}, \lambda_{2}, \beta\right) .
$$




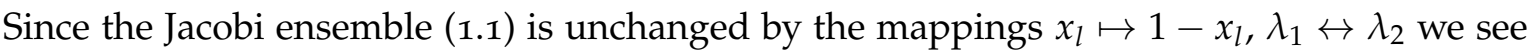
that

$$
E_{N}^{\mathrm{J}}\left(0 ;(s, 1) ; \lambda_{1}, \lambda_{2}, \beta\right)=E_{N}^{\mathrm{J}}\left(0 ;(0, s) ; \lambda_{2}, \lambda_{1}, \beta\right)
$$

and so the density function $p_{\min }^{(N)}\left(s ; \lambda_{1}, \lambda_{2}, \beta\right)$ for the distribution of the smallest eigenvalue is related to $p_{\max }^{(N)}$ by

$$
p_{\min }^{(N)}\left(s ; \lambda_{1}, \lambda_{2}, \beta\right)=p_{\max }^{(N)}\left(s ; \lambda_{2}, \lambda_{1}, \beta\right)
$$

Interest in $p_{\mathrm{max}}^{(N)}$, for the choices of $\left(\lambda_{1}, \lambda_{2}, \beta\right)$ as relevant to multivariate statistics, comes by way of Roy's largest root test [2, 41]. Taken literally, this applies to the combination of Wishart matrices $W_{1}^{-1} W_{2}$, where $W_{1}$ and $W_{2}$ have the interpretation of covariance matrices within and between classes respectively; see [31. \$2.2] for a clear discussion. For a recent work on the computation of $p_{\max }^{(N)}$, specific to the case $\beta=1$ as is of primary interest in multivariate statistics, see [10].

The interpretation of the Jacobi ensemble in terms of singular values of a sub-block of a Haar distributed unitary matrix gives motivation for knowledge of $p_{\min }^{(N)}$ [14]. Thus particular randomised algorithms in scientific computing make use of these blocks in linear systems, and so an important quantity is the corresponding condition number, which is controlled by the smallest eigenvalue.

In the general case, $p_{\min }^{(N)}$ and $p_{\max }^{(N)}$ represent examples of extreme value statistics in a strongly correlated system [34]. For large $N$, and with tuning of the parameters to a soft edge, large deviation deviation principles apply, giving rise to a macroscopic viewpoint of the tails [20, 35].

1.3. Our study. Our aim is to provide computable, structured expressions for

$$
E_{N}^{\mathrm{J}}\left(0 ;(s, 1) ; \lambda_{1}, \lambda_{2}, \beta\right)=\int_{0}^{s} d x_{1} \ldots \int_{0}^{s} d x_{N} \mathcal{P}^{\mathrm{J}}\left(x_{1}, \ldots, x_{N}\right)
$$

This will be done for the parameter ranges

(1) $\lambda_{1}>-1, \lambda_{2} \in \mathbb{Z}_{\geq 0}, \beta>0$;

(2) $\lambda_{1}>-1, \lambda_{2}=-\beta / 2+k>-1\left(k \in \mathbb{Z}_{\geq 0}\right), \beta>0$;

(3) $\lambda_{1} \in \mathbb{Z}_{\geq 0}, \lambda_{2}>-1, \beta \in \mathbb{Z}_{>0}$. 
The starting point for cases (1) and (2) is to change variables $x_{l} \mapsto s x_{l}$ in (1.9) and so obtain

$$
\begin{aligned}
& E_{N}^{\mathrm{J}}\left(0 ;(s, 1) ; \lambda_{1}, \lambda_{2}, \beta\right) \\
& \quad=\frac{s^{N\left(\lambda_{1}+1\right)+\beta N(N-1) / 2}}{J_{N, \lambda_{1}, \lambda_{2}, \beta}} \int_{0}^{1} d x_{1} \cdots \int_{0}^{1} d x_{N} \prod_{l=1}^{N} x_{l}^{\lambda_{1}}\left(1-s x_{l}\right)^{\lambda_{2}} \prod_{1 \leq j<k \leq N}\left|x_{k}-x_{j}\right|^{\beta} .
\end{aligned}
$$

In case (1), in which $\lambda_{2} \in \mathbb{Z}_{\geq 0}$, we see that the multiple integral in 1 .10 is a polynomial of degree $\lambda_{2} N$ and thus

$$
E_{N}^{\mathrm{J}}\left(0 ;(s, 1) ; \lambda_{1}, \lambda_{2}, \beta\right)=s^{N\left(\lambda_{1}+1\right)+\beta N(N-1) / 2} \sum_{p=0}^{\lambda_{2} N} \gamma_{p} s^{p},
$$

for certain coefficients $\left\{\gamma_{p}\right\}$. We will show that a known linear differential-difference equation [17] from the broader theory of the Selberg integral (see e.g. [19, Ch. 4]) can be used to compute these coefficients.

With $\lambda_{2}=-\beta / 2$ and thus in case (2), although the multiple integral in 1.10 is no longer a polynomial, it is known to equal a particular classical Gauss hypergeometric function [32, 14], [19, Prop. 13.1.3]

$$
\begin{aligned}
\frac{1}{J_{N, \lambda_{1}, 0, \beta}} \int_{0}^{1} d x_{1} \cdots \int_{0}^{1} d x_{N} \prod_{l=1}^{N} x_{l}^{\lambda_{1}}\left(1-s x_{l}\right)^{-\beta / 2} \prod_{1 \leq j<k \leq N}\left|x_{k}-x_{j}\right|^{\beta} \\
\quad={ }_{2} F_{1}\left(\beta N / 2,(\beta / 2)(N-1)+\lambda_{1}+1, \beta(N-1)+\lambda_{1}+2 ; s\right) .
\end{aligned}
$$

Using this as a seed in the differential-difference equation of [17] leads to the structured expression

$$
E_{N}^{\mathrm{J}}\left(0 ;(s, 1) ; \lambda_{1},-\beta / 2+k, \beta\right)=s^{N\left(\lambda_{1}+1\right)+\beta N(N-1) / 2}\left(P(s) f(s)+Q(s) f^{\prime}(s)\right),
$$

where $f(s)$ is the Gauss ${ }_{2} F_{1}$ function in 1 .12), and $P(s), Q(s)$ are polynomials of degree less than or equal to $k N, k(N+1)$. We remark that an analogous structure, applying to the probability density of the smallest eigenvalue density in the Laguerre orthogonal ensemble, for Laguerre weight $\chi_{x>0} x^{m-1 / 2} e^{-x}$ with $m \in \mathbb{Z}_{\geq 0}$, has been given by Edelman [15].

We now turn our attention to the parameter range specifying (3). We begin by using the symmetry of the integrand in (1.9) to order the integration variables

$$
s>x_{1}>x_{2}>\cdots>x_{N-1}>x_{N}>0 .
$$


Then

$$
\prod_{1 \leq j<k \leq N}\left|x_{k}-x_{j}\right|^{\beta}=\prod_{1 \leq j<k \leq N}\left(x_{j}-x_{k}\right)^{\beta}=\sum_{\kappa} \alpha_{\kappa} \prod_{l=1}^{N} x_{l}^{\kappa_{l}},
$$

for some coefficients $\alpha_{\kappa}$, where $\kappa$ is a partition $\kappa_{1} \geq \kappa_{2} \geq \cdots \geq \kappa_{N} \geq 0$ involving up to $N$ parts of fixed length $\sum_{j=1}^{N} \kappa_{i}=\beta N(N-1) / 2$, and further constrained so that $\kappa_{j} \leq(N-j) \beta$.

Substituting in $(1.9)$ shows that for $\beta \in \mathbb{Z}_{\geq 0}$

$$
E_{N}^{\mathrm{J}}\left(0 ;(s, 1) ; \lambda_{1}, \lambda_{2}, \beta\right)=\frac{N !}{J_{N, \lambda_{1}, \lambda_{2}, \beta}} \sum_{\kappa} \alpha_{\kappa} \int_{0}^{s} d x_{1} \int_{0}^{x_{1}} d x_{2} \cdots \int_{0}^{x_{N-1}} d x_{N} \prod_{l=1}^{N} x_{l}^{\lambda_{1}+\kappa_{l}}\left(1-x_{l}\right)^{\lambda_{2}} .
$$

In general,

$$
\int_{0}^{x} d X X^{a}(1-X)^{b}=\int_{0}^{1} d X X^{a}(1-X)^{b}-\int_{0}^{1-x} d X X^{b}(1-X)^{a} .
$$

For $a$ a non-negative integer, the final factor in the second integral can be expanded according to the binomial theorem, telling us that in this circumstance

$$
\int_{0}^{x} d X X^{a}(1-X)^{b}=B(a, b)-\sum_{p=0}^{a}(-1)^{p} C_{p}^{a} \frac{1}{b+p+1}(1-x)^{b+p+1},
$$

where $C_{p}^{a}$ denotes the binomial coefficient, and

$$
B(a, b):=\int_{0}^{1} d X X^{a}(1-X)^{b}=\frac{\Gamma(a+1) \Gamma(b+1)}{\Gamma(a+b+2)}
$$

is the Euler beta function.

Making repeated use of 1.16 in 1.15 shows that for the parameter range of (3)

$$
E_{N}^{\mathrm{J}}\left(0 ;(s, 1) ; \lambda_{1}, \lambda_{2}, \beta\right)=1+\sum_{q=1}^{N}(1-s)^{q\left(\lambda_{2}+1\right)} \sum_{l=l_{\min }(q)}^{l_{\max }(q)} \gamma_{q, l}(1-s)^{l}
$$

for some $\left\{\gamma_{q, l}\right\}$, and where $l_{\min }(q), l_{\max }(q)$ are shown below to be given by

$$
l_{\min }(q)=q(q-1) \beta / 2, \quad l_{\max }(q)=q \lambda_{1}+q(N-q) \beta+q(q-1) \beta / 2,
$$

allowing 1.18 to be rewritten

$$
E_{N}^{\mathrm{J}}\left(0 ;(s, 1) ; \lambda_{1}, \lambda_{2}, \beta\right)=1+\sum_{q=1}^{N}(1-s)^{q\left(\lambda_{2}+1\right)+q(q-1) \beta / 2} \sum_{l=0}^{q \lambda_{1}+q(N-q) \beta} \tilde{\gamma}_{q, l}(1-s)^{l} .
$$

We will show how the differential-difference equation of [17] can be adapted to compute $\left\{\tilde{\gamma}_{q, k}\right\}$. In fact two methods are given, with the second involving the recursive computation of each of $\left\{E_{n}^{\mathrm{J}}\left(0 ;(s, 1), \lambda_{1}, \lambda_{2}, \beta\right)\right\}_{n=1}^{N}$, given $E_{1}^{\mathrm{J}}$ as the initial condition. We will show in 
Appendix A how this latter method can be modified to provide an analogous recursive computational scheme for the gap probability $E_{N}^{C}(0 ;(0, \phi) ; \beta)$, the probability of no eigenvalues in the $\beta$-circular ensemble, specified by the class of eigenvalue probability density functions

$$
\frac{1}{\mathcal{N}_{\beta, N}} \prod_{1 \leq j<k \leq N}\left|e^{i \theta_{k}}-e^{i \theta_{j}}\right|^{\beta}
$$

Here $0 \leq \theta_{j} \leq 2 \pi,(j=1, \ldots, N)$ and $\mathcal{N}_{\beta, N}$ is the normalisation; see e.g. [19]. Prop. 4.7.2] for its explicit evaluation.

\section{Computation OF THE COEFFicients}

2.1. The differential-difference system. Our ability to compute the coefficients in the structural forms (1.11), 1.13 and (1.18) rests with a recursion scheme satisfied by a generalisation of the multi-dimensional integral in (1.10).

Let $e_{p}\left(y_{1}, \ldots, y_{N}\right)$ denote the elementary symmetric polynomials in $\left\{y_{j}\right\}_{j=1}^{N}$, and define

$$
\begin{aligned}
J_{p, N, R}^{(\alpha)}(x)=\frac{1}{C_{p}^{N}} \int_{R} d t_{1} \cdots \int_{R} d t_{N} \prod_{l=1}^{N} t_{l}^{\lambda_{1}}(1- & \left.t_{l}\right)^{\lambda_{2}}\left(x-t_{l}\right)^{\alpha} \\
& \times \prod_{1 \leq j<k \leq N}\left|t_{k}-t_{j}\right|^{\beta} e_{p}\left(x-t_{1}, \ldots, x-t_{N}\right)
\end{aligned}
$$

where $R=[0,1]$ or $R=[x, 1]$. This family of multiple integrals satisfies the differentialdifference system [17], [19, \$4.6.4 ] , later observed to be equivalent to a certain Fuchsian matrix differential equation [26],

$$
(N-p) E_{p} J_{p+1}(x)=\left(A_{p} x+B_{p}\right) J_{p}(x)-x(x-1) \frac{d}{d x} J_{p}(x)+D_{p} x(x-1) J_{p-1}(x),
$$

where we have abbreviated $J_{p, N, R}^{(\alpha)}(x)=: J_{p}(x)$, and

$$
\begin{aligned}
& A_{p}=(N-p)\left(\lambda_{1}+\lambda_{2}+\beta(N-p-1)+2(\alpha+1)\right) \\
& B_{p}=(p-N)\left(\lambda_{1}+\alpha+1+(\beta / 2)(N-p-1)\right) \\
& D_{p}=p((\beta / 2)(N-p)+\alpha+1) \\
& E_{p}=\lambda_{1}+\lambda_{2}+1+(\beta / 2)(2 N-p-2)+(\alpha+1) .
\end{aligned}
$$

\footnotetext{
${ }^{1}$ These references have $\alpha$ replaced by $\alpha-1$ relative to our 2.1 .
} 
COMPUTABLE STRUCTURAL FORMULAS FOR THE DISTRIBUTION OF THE $\beta$-JACOBI EDGE EIGENVALUES 9

Starting with knowledge of $\left.J_{p, N, R}^{(\alpha)}(x)\right|_{p=0}$, application of the recurrence for $p=0,1, \ldots, N$ gives us an expression for $J_{0, N, R}^{(\alpha+1)}(x)$, since from the definition 2.1)

$$
\left.J_{p, N, R}^{(\alpha)}(x)\right|_{p=N}=J_{0, N, R}^{(\alpha+1)}(x) .
$$

Repeating this, we can deduce an expression for $J_{0, N, R}^{(\alpha+k)}(x)$ from knowledge of $J_{0, N, R}^{(\alpha)}(x)$ for any positive integer $k$.

2.2. Cases (1) and (2). For application to the parameter ranges (1) and (2) as listed below 1.9) we set $R=[0,1]$ in (2.1) and consider the transformed integrals

$$
\begin{aligned}
& \text { (2.4) } \tilde{J}_{p, N}^{(\alpha)}(x)=\left.\frac{x^{N \alpha+p}}{J_{N, \lambda_{1}, \lambda_{2}, \beta}} J_{p, N, R}^{(\alpha)}(1 / x)\right|_{R=[0,1]} \\
& =\frac{1}{J_{N, \lambda_{1}, \lambda_{2}, \beta} C_{p}^{N}} \int_{0}^{1} d t_{1} \cdots \int_{0}^{1} d t_{N} \prod_{l=1}^{N} t_{l}^{\lambda_{1}}\left(1-t_{l}\right)^{\lambda_{2}}\left(1-x t_{l}\right)^{\alpha} \\
& \times \prod_{1 \leq j<k \leq N}\left|t_{k}-t_{j}\right|^{\beta} e_{p}\left(1-x t_{1}, \ldots, 1-x t_{N}\right) .
\end{aligned}
$$

The normalisations are such

$$
\left.\tilde{J}_{p, N}^{(\alpha)}(x)\right|_{x=0}=\left.\tilde{J}_{p, N}^{(\alpha)}(x)\right|_{p=\alpha=0}=1
$$

and we have, using (1.10,

$$
E_{N}^{\mathrm{J}}\left(0 ;(s, 1) ; \lambda_{1}, \lambda_{2}, \beta\right)=\left.s^{N\left(\lambda_{1}+1\right)+\beta N(N-1) / 2} \frac{J_{N, \lambda_{1}, 0, \beta}}{J_{N, \lambda_{1}, \lambda_{2}, \beta}} \cdot \tilde{J}_{0, N}^{(\alpha)}(s)\right|_{\lambda_{2}=0, \alpha \mapsto \lambda_{2}} .
$$

In addition to the closed form evaluation (1.12) as is relevant to case (2), we also have that [3], [19, Prop. 13.1.2]

$$
\text { (2.7) } \begin{aligned}
\left.\tilde{J}_{p, N}^{(\alpha)}(x)\right|_{p=0, \lambda_{2}=0, \alpha=1} \\
\quad={ }_{2} F_{1}\left(-N,-(N-1)-(2 / \beta)\left(\lambda_{1}+1\right),-2(N-1)-(2 / \beta)\left(\lambda_{1}+2\right) ; x\right),
\end{aligned}
$$

which is relevant to case (1).

In terms of the transformed integrals (2.4), and with the further abbreviation of notation $\tilde{J}_{p, N}^{(\alpha)}(x)=: \tilde{J}_{p}(x)$ analogous to what was used in 2.2 , the differential-difference system 2.2 reads

$$
\begin{aligned}
& (N-p) E_{p} \tilde{J}_{p+1}(x) \\
& =\left(A_{p}-(N \alpha+p)+\left(B_{p}+N \alpha+p\right) x\right) \tilde{J}_{p}(x)+x(1-x) \frac{d}{d x} \tilde{J}_{p}(x)+D_{p}(1-x) \tilde{J}_{p-1}(x) .
\end{aligned}
$$


In case (1), the recurrence is begun with $\lambda_{2}=0, \alpha=0$ using the second of the equations in (2.5) which is the $\lambda_{2}=0$ parameter value in (1). After iterating for $p=0,1, \ldots, N$, use is made of the analogue of (2.3) to now have at hand the form of the polynomial $\left.\tilde{J}_{0, N}^{(\alpha)}(x)\right|_{\lambda_{2}=0, \alpha=1}$ (which must agree with 2.7$)$ ). This procedure is then repeated a total of $\lambda_{2}$ times to eventually compute $\left.\tilde{J}_{0, N}^{(\alpha)}(x)\right|_{\lambda_{2}=0, \alpha \mapsto \lambda_{2}}$. After multiplication by a suitable normalisation, as made explicit in (2.6), we have available all the coefficients $\left\{\gamma_{p}\right\}$ in (1.11. Note that since $\left.E_{N}^{\mathrm{J}}\left(0 ;(s, 1) ; \lambda_{1}, \lambda_{2}, \beta\right)\right|_{s=1}=1$, we must have the sum rule

$$
\sum_{p=0}^{N \lambda_{2}} \gamma_{p}=1,
$$

which provides a useful check on the computation. Another check follows from the fact that in addition to 2.2), the family of integrals 2.1 in the polynomial case $R=[0,1]$, $\alpha \in \mathbb{Z}_{\geq 0}$ also satisfy a multidimensional difference equation [22, Eq. (30)], which allows for an independent computation.

We now turn our attention to case (2). First, we will show how (2.8), initialised with $\alpha=-\beta / 2$ so that $\tilde{J}_{0}$ is given by 1.12 , implies the structure 1.13 . An essential point is that generally the Gauss hypergeometric function ${ }_{2} F_{1}(a, b, c ; x)$ satisfies the second order linear differential equation

$$
x(1-x) \frac{d^{2} y}{d x^{2}}+(c-(a+b+1) x) \frac{d y}{d x}-a b y=0 .
$$

Denote the Gauss hypergeometric function in 1.12 by $f(a, b, c ; s)=: f(s)$ as is consistent with the notation used in 1.13 . For some functions $P_{p}, Q_{p}$ write

$$
\tilde{J}_{p}(x)=P_{p}(x) f(x)+Q_{p}(x) f^{\prime}(x) .
$$

Substituting in 2.8 and making use of 2.10 shows

$$
\begin{aligned}
(N-p) E_{p} P_{p+1}(x)=\left(A_{p}-(N \alpha\right. & \left.+p)+\left(B_{p}+N \alpha+p\right) x\right) P_{p}(x) \\
& +D_{p}(1-x) P_{p-1}(x)+x(1-x) P_{p}^{\prime}(x)+a b Q_{p}(x)
\end{aligned}
$$

and

$$
\begin{aligned}
& (N-p) E_{p} Q_{p+1}(x) \\
& =\left(A_{p}-(N \alpha+p)+\left(B_{p}+N \alpha+p\right) x\right) Q_{p}(x)+D_{p}(1-x) Q_{p-1}(x) \\
& +x(1-x)\left(P_{p}(x)+Q_{p}^{\prime}(x)\right)-(c-(a+b+1) x) Q_{p}(x) .
\end{aligned}
$$

Now (1.12) and (2.4) tells us that for $\alpha=-\beta / 2, \lambda_{2}=0$ we have $\left(P_{0}(x), Q_{0}(x)\right)=(1,0)$, so we see by iteration of 2.12 and 2.13 that 1.13 holds true, with $P(s), Q(s)$ polynomials 
of the stated degree. The recursion is initialised with $\left.\tilde{J}_{0}^{(\alpha)}\right|_{\lambda_{2}=0, \alpha=-\beta / 2}$ and then 2.12 and 2.13) are iterated for $p=0,1, \ldots, N$ to obtain $\left.\tilde{J}_{N}^{(\alpha)}\right|_{\lambda_{2}=0, \alpha=-\beta / 2}$. The latter is identical to $\left.\tilde{J}_{0}^{(\alpha)}\right|_{\lambda_{2}=0, \alpha=-\beta / 2+1}$, thereby resulting in the increase of $\alpha$ by 1 . This procedure is repeated $k$ times to eventually obtain $\left.\tilde{J}_{N}^{(\alpha)}\right|_{\lambda_{2}=0, \alpha=-\beta / 2+k}$, which in turn results in the desired gap probability expression using (2.6.).

A number of checks on the computation are possible. One is that

$$
\lim _{s \rightarrow 1^{-}}\left(P(s) f(s)+Q(s) f^{\prime}(s)\right)=1,
$$

as analogous to (2.9). Moreover, for $\beta$ even, and $k$ a positive integer such that $-\beta / 2+k \geq 0$, we see that case (2) coincides with case (1), and in particular the structure (1.11) must hold true with $\lambda_{2}=-\beta / 2+k$.

In figures 2.1 and 2.2, we show gap probabilities for the cases (1) and (2) obtained using the recursive schemes described above. We consider three sets of parameters for each of these cases. Moreover, for comparison, we also show the results obtained using the Monte Carlo simulations of the matrix model (1.7) as overlaid symbols and agree perfectly with the analytical results depicted using solid curves. A Mathematica [47] file with codes implementing the above recursive schemes is provided as an ancillary file.

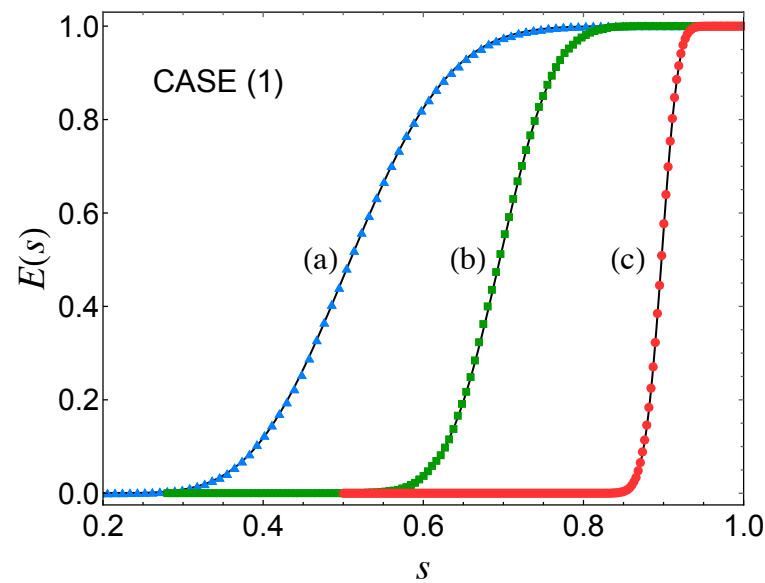

Figure 2.1. Gap probabilities $E_{N}^{\mathrm{J}}\left(0 ;(s, 1) ; \lambda_{1}, \lambda_{2}, \beta\right) \equiv E(s)$ in case (1) for three sets of parameter values: (a) $n=7, \lambda_{1}=-3 / 4, \lambda_{2}=9, \beta=7 / 8$; (b) $n=10, \lambda_{1}=2, \lambda_{2}=15, \beta=3 / 2$; (c) $n=15, \lambda_{1}=5, \lambda_{2}=25, \beta=5$. The solid curves have been obtained using the recursive scheme and symbols are based on Monte Carlo simulation of matrix model (1.7). 


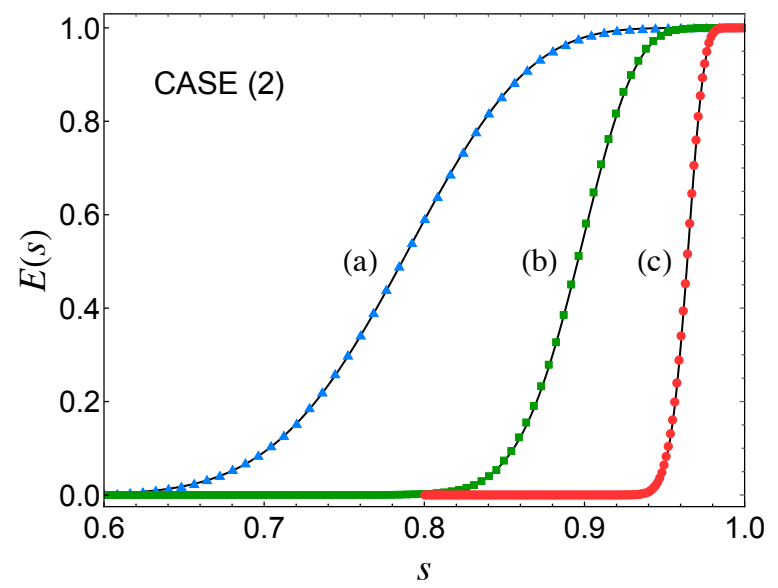

FIGURE 2.2. Gap probabilities $E_{N}^{\mathrm{J}}\left(0 ;(s, 1) ; \lambda_{1}, \lambda_{2}, \beta\right) \equiv E(s)$ in case (2) for three sets of parameter values: (a) $n=5, \beta=1 / 2, \lambda_{1}=9, k=6, \lambda_{2}=$ $-\beta / 2+k=23 / 4$; (b) $n=9, \beta=8 / 3, \lambda_{1}=7, k=10, \lambda_{2}=-\beta / 2+k=26 / 3$; (c) $n=18, \beta=5, \lambda_{1}=16 / 3, k=16, \lambda_{2}=-\beta / 2+k=27 / 2$.

2.3. Case (3). We now deal with the parameter range (3) as listed below (1.9) and, to begin with, we focus on even $\beta$. Eventually, we will discuss a strategy to handle a general $\beta \in \mathbb{Z}_{>0}$. For the present case, we require the family of integrals 2.1 with both $R=[0,1]$ and $R=[x, 1]$ :

$$
\int_{R} d X \mapsto\left(\int_{0}^{1}+\zeta \int_{x}^{1}\right) d X
$$

This choice of $R=R(\zeta)$ relates to $E_{N}^{\mathrm{J}}\left(0 ;(s, 1) ; \lambda_{1}, \lambda_{2}, \beta\right)$ according to

$$
E_{N}^{\mathrm{J}}\left(0 ;(s, 1) ; \lambda_{1}, \lambda_{2}, \beta\right)=\left.\frac{1}{J_{N, \lambda_{1}, \lambda_{2}, \beta}} J_{0, N, R(\zeta)}^{(\alpha)}(x)\right|_{\alpha=0, \zeta=-1} .
$$

The relevance of introducing this seemingly complicated choice of $R$ comes from a viewpoint of the differential-difference system 2.2 as a matrix linear differential equation [26]. Thus with $R(\zeta)$ as specified by 2.15), we read off from 2.2 that the vector of integrals

$$
\mathbf{J}_{N}^{(\alpha)}(x ; \zeta)=\frac{1}{J_{N, \lambda_{1}, \lambda_{2}, \beta}}\left[J_{p, N, R(\zeta)}^{(\alpha)}(x)\right]_{p=0}^{N}
$$

satisfies the matrix differential equation

$$
\frac{d}{d x} \mathbf{J}=\left(\frac{\mathbf{Z}_{-1}}{x-1}+\frac{\mathbf{Z}_{0}}{x}+\mathbf{Y}\right) \mathbf{J}
$$


COMPUTABLE STRUCTURAL FORMULAS FOR THE DISTRIBUTION OF THE $\beta$-JACOBI EDGE EIGENVALUES 13 where $\mathbf{Z}_{-1}, \mathbf{Z}_{0}, \mathbf{Y}$ are $(N+1) \times(N+1)$ matrices specified by

$$
\begin{aligned}
\mathbf{Z}_{-1} & =\operatorname{diag}\left[A_{p}+B_{p}\right]_{p=0}^{N}-\operatorname{diag}^{+}\left[(N-p) E_{p}\right]_{p=0}^{N-1} \\
\mathbf{Z}_{0} & =\operatorname{diag}\left[-B_{p}\right]_{p=0}^{N}+\operatorname{diag}^{+}\left[(N-p) E_{p}\right]_{p=0}^{N-1} \\
\mathbf{Y} & =\operatorname{diag}^{-}\left[D_{p}\right]_{p=1}^{N} .
\end{aligned}
$$

Here diag ${ }^{+}$refers to the first diagonal above the main diagonal, and diag ${ }^{-}$the first diagonal below; all other entries are zero.

The matrix differential equation (2.18) admits Frobenius type solutions

$$
\left[\mathbf{w}_{p}^{(q)}(x)\right]_{p=0}^{N}, \quad \mathbf{w}_{p}^{(q)}(x):=(1-x)^{\mu_{q}}\left[\sum_{l=0}^{\infty} c_{l, p}^{(q)}(1-x)^{l}\right]_{p=0}^{N},
$$

where $\mu_{q}=\left(A_{q}+B_{q}\right)=(N-q)\left(\lambda_{2}+(\beta / 2)(N-q-1)+1\right)$. Let us write $\mathbf{c}_{l}^{(q)}=\left[c_{l, p}^{(q)}\right]_{p=0}^{N}$. Substituting 2.19) in 2.18), expanding both sides in a power series in $(1-x)$, and equating like coefficients shows

$$
\left(\mathbf{Z}_{-1}-\left(\mu_{q}+n\right) \mathbb{I}_{N+1}\right) \mathbf{c}_{n}^{(q)}=\left(\mathbf{Z}_{0}+\mathbf{Y}\right) \mathbf{c}_{n-1}^{(q)}+\mathbf{Z}_{0}\left(\sum_{s=1}^{n-1} \mathbf{c}_{n-1-s}^{(q)}\right)
$$

valid for $n \geq 1$. For $n=0$ the right hand side is zero, telling us that $\mathbf{c}_{0}^{(q)}$ is the eigenvector of $\mathbf{Z}_{-1}$ with eigenvalue $A_{q}+B_{q}$. Only the first $q$ components are non-zero, and satisfy the recurrence

$$
c_{0, p+1}^{(q)}=\frac{A_{p}+B_{p}-A_{q}-B_{q}}{(N-p) E_{p}} c_{0, p^{\prime}}^{(q)} \quad(p=0, \ldots, q-1),
$$

where we are free to choose

$$
c_{0,0}^{(q)}=1
$$

With this as the initial condition, we can use 2.20 to recursively compute the coefficients in 2.19), provided $\mathbf{Z}_{-1}-\left(\mu_{q}+n\right) \mathbb{I}_{N+1}$ is invertible for $n \geq 1$; for this latter requirement it is sufficient that $\lambda_{2}$ be a generic parameter. Moreover, for the $\lambda_{2}$ values which result in a singular $\mathbf{Z}_{-1}-\left(\mu_{q}+n\right) \mathbb{I}_{N+1}$, the recursion scheme may be implemented with $\lambda_{2}$ kept as a variable. The correct result can then be produced from the final expression by assigning $\lambda_{2}$ the desired value. However, this does slow down the recursive scheme considerably, if the parameter values are large. Therefore, in such situations, it is generally better to implement an alternative recursion scheme, as discussed ahead. 
With $\left[\zeta^{q}\right] f$ denoting the coefficient of $\zeta^{q}$ in $f$, from the definitions 2.1 and 2.15

$$
\begin{aligned}
{\left.\left[\zeta^{q}\right] J_{p, N, R(\zeta)}^{(\alpha)}(x)\right|_{\alpha=0}=\frac{C_{q}^{N}}{C_{p}^{N}} } & \int_{x}^{1} d t_{1} \cdots \int_{x}^{1} d t_{q} \int_{0}^{1} d t_{q+1} \cdots \int_{0}^{1} d t_{N} \\
& \times \prod_{l=1}^{N} t_{l}^{\lambda_{1}}\left(1-t_{l}\right)^{\lambda_{2}} \prod_{1 \leq j<k \leq N}\left|t_{k}-t_{j}\right|^{\beta} e_{p}\left(x-t_{1}, \ldots, x-t_{N}\right) .
\end{aligned}
$$

According to 2.16 ,

$$
E_{N}^{\mathrm{J}}\left(0 ;(s, 1) ; \lambda_{1}, \lambda_{2}, \beta\right)=\left.\frac{1}{J_{N, \lambda_{1}, \lambda_{2}, \beta}} \sum_{q=0}^{N}(-1)^{q}\left[\zeta^{q}\right] J_{p, N, R(\zeta)}^{(\alpha)}(x)\right|_{\alpha=p=0} .
$$

We know that independent of $q$, the family of multiple integrals 2.23 satisfies the matrix differential equation (2.18). Moreover a simple change of variables shows that for $\beta$ a non-negative even integer, the power series in $(1-x)$ has the structure 2.19$)$ with $q$ replaced by $N-q$, and furthermore $\left.c_{l, p}^{(N-q)}\right|_{p=0}=0$ for $l>l_{\max }(q)$, where $l_{\max }(q)$ is given by 1.19$)$, thus providing justification of the latter, and also that $\left.c_{l, p}^{(N-q)}\right|_{p=0}=0$ for $l<l_{\min }(q)$, where

$$
l_{\min }(q)=q(q-1) \beta / 2 \text {. }
$$

In particular, it must be that 2.23 is proportional to 2.19) with $q$ replaced by $N-q$. If we normalise 2.19) so that $c_{0,0}^{(N-q)}=1$, consideration of the small $x$ behaviour of the multiple integral corresponding to $\left.J_{p, N, R(\zeta)}^{(\alpha)}(x)\right|_{\alpha=0, p=0}$ allows us to compute the proportionality and so conclude

$$
\left.\left[\zeta^{q}\right] \mathbf{J}_{N}^{(\alpha)}(x ; \zeta)\right|_{\alpha=0}=C_{q}^{N} \frac{J_{N-q, \lambda_{2}+q \beta, \beta} J_{q, \lambda_{2}, 0, \beta}}{J_{N, \lambda_{1}, \lambda_{2}, \beta}}\left[\mathbf{w}_{p}^{(N-q)}(x)\right]_{p=0}^{N} .
$$

This holds true for general $\lambda_{1}>-1$; the significance of restricting to $\lambda_{1}$ a non-negative integer is that then the power series in (2.19) terminates. We remark that in the case $\beta=2$ an alternative way to compute the expansion 1.18 is to use the characterisation of $E_{N}^{\mathrm{J}}$ in that case in terms of the solution of a particular Painlevé VI equation in sigma form; see [19] \$8.3.1 and eq. (8.77)]. Working overlapping with the above in the special case $\beta=1$ can also be found in earlier literature. Specifically, a matrix differential equation equivalent to (2.18) for the corresponding family of integrals [2.23) was derived by Davis [12] in a pioneering paper on this characterisation published in 1972.

There is an alternative method that can be used for the same purposes of computing the expansion (2.19) and to handle $\beta \in \mathbb{Z}_{>0}$. This is to follow a procedure detailed in our recent work [23] for the $\beta$-Laguerre ensemble, which itself can be traced back to [29] where it was used to give a recursive computation of the probability density function for the Gaussian orthogonal ensemble; for the recursion corresponding to 2.2 for the $\beta$-Laguerre ensemble 
see [33], [25]. For $\lambda_{1}$ a non-negative integer, and general $\lambda_{2}>-1$, we begin with 1.16) to compute $\int_{0}^{s} d t_{1} t_{1}^{\lambda_{1}}\left(1-t_{1}\right)^{\lambda_{2}}$ in the required form. Applying the recurrence 2.2) with $\alpha=0, \ldots, \beta-1$ gives the evaluation of

$$
\int_{0}^{s} d t_{1} t_{1}^{\lambda_{1}}\left(1-t_{1}\right)^{\lambda_{2}}\left(s-t_{1}\right)^{\beta}
$$

with the structure 1.16 now replaced by $Q(s)+(1-s)^{\lambda_{2}} R(s)$ for polynomials $Q(s), R(s)$ in $(1-s)$. In this we set $s=t_{2}$, multiply by $\left(1-t_{2}\right)^{\lambda_{2}} t_{2}^{\lambda_{1}}$, and integrate over $t_{2}$ from 0 to $s$ using 1.16) again, thereby evaluating $E_{N}^{\mathrm{J}}\left(0 ;(s, 1) ; \lambda_{1}, \lambda_{2}, \beta\right)$ for $N=2$ with the structure (1.18). Repeating this procedure, we see that for the parameter range (3) we can compute $E_{N}^{J}\left(0 ;(s, 1) ; \lambda_{1}, \lambda_{2}, \beta\right)$ for any fixed $N$ from knowledge of $E_{N-1}^{J}\left(0 ;(s, 1) ; \lambda_{1}, \lambda_{2}, \beta\right)$, and moreover the final expression will have the structure (1.18).

A sum rule associated with 1.18 is to use the fact that $\left.E_{N}^{J}\left(0 ;(s, 1) ; \lambda_{1}, \lambda_{2}, \beta\right)\right|_{s=0}=0$ to conclude

$$
0=1+\sum_{q=1}^{N} \sum_{l=0}^{q \lambda_{1}+q(N-q) \beta} \tilde{\gamma}_{q, l} .
$$

Also, for $\lambda_{2}=-\beta / 2+k>-1$, agreement must be obtained with case (3), and for $\lambda_{2} \in \mathbb{Z}_{\geq 0}$ when (2.12) is a polynomial in $s$, the result must agree with that obtained in case (1).

In figure 2.3. we show the gap probabilities obtained using the above described recursive approaches in the case (3) for three sets of parameter values. The first two sets can be handled by the recursion scheme involving matrices given by (2.20), whereas for the last one, we require the alternative recursive approach. As for figures 2.1 and 2.2. Monte Carlo simulation results based on (1.7), shown as overlaid symbols, are found to be in perfect agreement with the solid curves obtained using the recursive schemes. Mathematica [47] files with codes implementing the above described recursion schemes are attached as ancillary files.

2.4. Direct computation of $p_{\max }^{(N)}$. Our concern in the above sections has been with the computation of $E_{N}^{\mathrm{J}}\left(0,(s, 1) ; \lambda_{1}, \lambda_{2}, \beta\right)$. The probability density function $p_{\max }^{(N)}$ for the distribution of the largest eigenvalue is then computed by differentiation according to (1.8). Here we consider the task of computing $p_{\max }^{(N)}$ directly for the ranges of parameters (1) and (2) as listed below (1.9).

Differentiating (1.9) gives

$$
p_{\max }^{(N)}\left(s, \lambda_{1}, \lambda_{2}, \beta\right)=N \int_{0}^{s} d x_{2} \cdots \int_{0}^{s} d x_{N} \mathcal{P}^{\mathrm{J}}\left(s, x_{2}, \ldots, x_{N}\right) .
$$




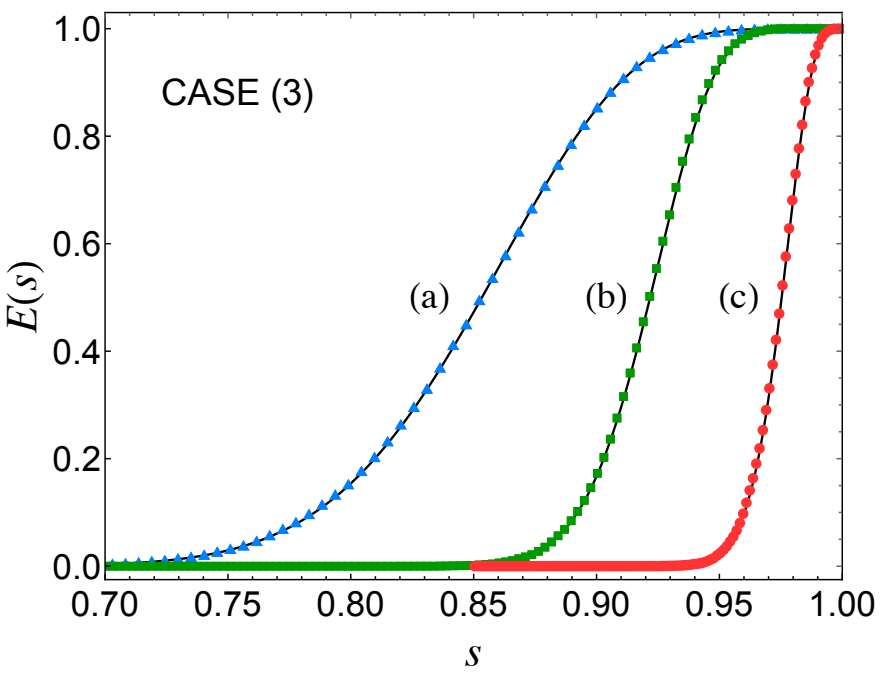

FIGURE 2.3. Gap probabilities $E_{N}^{\mathrm{J}}\left(0 ;(s, 1) ; \lambda_{1}, \lambda_{2}, \beta\right) \equiv E(s)$ in case (3) for three sets of parameter values: (a) $n=6, \lambda_{1}=5, \lambda_{2}=17 / 3, \beta=2$; (b) $n=11, \lambda_{1}=1, \lambda_{2}=49 / 5, \beta=4$; (c) $n=25, \lambda_{1}=4, \lambda_{2}=32 / 9, \beta=1$.

Changing variables $x_{l} \mapsto s x_{l}$ shows that analogous to 1.10

$$
\begin{aligned}
p_{\max }^{(N)}\left(s ; \lambda_{1}, \lambda_{2}, \beta\right) & =\frac{N s^{N\left(\lambda_{1}+1\right)+\beta N(N-1) / 2-1}(1-s)^{\lambda_{2}}}{J_{N, \lambda_{1}, \lambda_{2}, \beta}} \\
& \times \int_{0}^{1} d x_{2} \cdots \int_{0}^{1} d x_{N} \prod_{l=2}^{N} x_{l}^{\lambda_{1}}\left(1-x_{l}\right)^{\beta}\left(1-s x_{l}\right)^{\lambda_{2}} \prod_{2 \leq j<k \leq N}\left|x_{k}-x_{j}\right|^{\beta} .
\end{aligned}
$$

It follows that for $\lambda_{2} \in \mathbb{Z}_{\geq 0}$, which is case (I) of the parameter ranges, we have the structure

$$
p_{\max }^{(N)}\left(s ; \lambda_{1}, \lambda_{2}, \beta\right)=s^{N\left(\lambda_{1}+1\right)+\beta N(N-1) / 2-1}(1-s)^{\lambda_{2}} \sum_{p=0}^{\left(\lambda_{2}-1\right) N} \tilde{\gamma}_{p} s^{p} .
$$

Note that this analytic form is consistent with differentiating 1.11 with respect to $s$. By appropriate choice of the parameters the recurrence 2.8 can be applied to directly compute $\left\{\tilde{\gamma}_{p}\right\}$ in 2.28.

For the parameter range (2), we require a modification of (1.12),

$$
\begin{aligned}
\frac{1}{J_{N-1, \lambda_{1}, \beta, \beta}} \int_{0}^{1} d x_{2} & \cdots \int_{0}^{1} d x_{N} \prod_{l=2}^{N} x_{l}^{\lambda_{1}}\left(1-s x_{l}\right)^{-\beta / 2} \prod_{2 \leq j<k \leq N}\left|x_{k}-x_{j}\right|^{\beta} \\
& ={ }_{2} F_{1}\left(\beta(N-1) / 2,(\beta / 2)(N-2)+\lambda_{1}+1, \beta(N-1)+\lambda_{1}+2 ; s\right)
\end{aligned}
$$


see e.g. [19, Prop. 13.1.3]. Using this as a seed in the recurrence (2.8), the method of the paragraph including $(2.10)$ allows us to compute polynomials $\tilde{P}(s), \tilde{Q}(s)$ such that

$$
p_{\max }^{(N)}\left(s ; \lambda_{1}, \lambda_{2}, \beta\right)=s^{N\left(\lambda_{1}+1\right)+\beta N(N-1) / 2-1}(1-s)^{\lambda_{2}}\left(\tilde{P}(s) \tilde{f}(s)+\tilde{Q}(s) \tilde{f}^{\prime}(s)\right),
$$

where $\tilde{f}(s)$ denotes the Gauss hypergeometric function in 2.29 .

2.5. The large $N$ limit. The distributions $p_{\max }^{(N)}$ and $p_{\min }^{(N)}$ permit two distinct large $N$ limits. One is to a hard edge state, which is dependent on the respective hard edge parameter: $\lambda_{2}$ for the largest eigenvalue, replaced by $\lambda_{1}$ for the smallest eigenvalue. As an explicit example, we see from 2.27 that

$$
\left.\lim _{N \rightarrow \infty} \frac{1}{N^{2}} p_{\max }^{(N)}\left(\left(1-\frac{s}{N^{2}}\right) ; \lambda_{1}, \lambda_{2}, \beta\right)\right|_{\lambda_{2}=0}=\frac{2}{\beta} e^{-\beta s / 2}
$$

However the dependence on $\lambda_{2}$ cannot be made explicit in general. Exceptions are for $\beta=1,2$ and 4 when the limiting hard edge distributions can be characterised in terms of both Painlevé transcendents and Fredholm determinants; see e..g. [19, §9.5.1, §9.8]. The Fredholm form is particularly well suited for numerical computations [9]. For positive integer and half integer $(\geq-1 / 2)$ values of $\lambda_{2}$ in the case $\beta=1$ there is a Pfaffian form [ 1 , and similarly for $\lambda_{2}$ even in the case $\beta=4$ [42]. The limiting structure is even simpler for $\lambda_{2}$ a positive integer, when it can be expressed as a determinant [21, 39].

In the circumstance that $\lambda_{2}$ is proportional to $N$, the limiting support of the eigenvalue density is bounded away from 1 . An appropriate scaling [30, 28] gives a well defined limiting distribution, corresponding to what is termed a soft edge state. This limiting distribution is independent of $\lambda_{1}, \lambda_{2}$. For $\beta=1,2$ and 4 it can be characterised in terms of Painlevé transcendents and Fredholm determinants [46]. An analogous, but more complicated characterisation is also known for $\beta=6$ [44].

A future application of our recursive, finite $N$, computations of the present paper is in relation to the theme of scaling this variables so as to obtain an optimal rate of convergence to the limiting distributions. In special cases, these scalings are quantified in [39] for the hard edge limit, and in [30] for the soft edge limit. In the recent works [24, 25], recursions applying for the $\beta$-Laguerre ensemble have been used to exhibit the optimal rates beyond the classical cases $\beta=1,2$ and 4 .

\section{ACKNOWLEDGEMENTS}

P.J.F. acknowledges support from the Australian Research Council (ARC) through the ARC Centre of Excellence for Mathematical \& Statistical Frontiers. 


\section{APPENDIX}

The $\beta$-circular ensemble 1.21 is a particular case of the (generalised) circular Jacobi $\beta$-ensemble. The latter is specified by the family of probability density functions on the unit circle proportional to

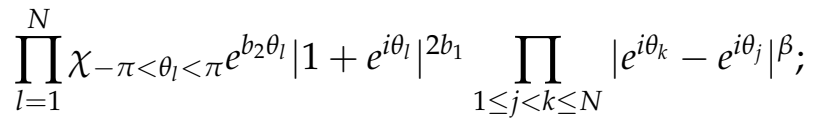

thus we set $b_{1}=b_{2}=0$. Introducing $\xi_{l}=e^{i \theta_{l}}(l=1, \ldots, N)$, and temporarily requiring that $b_{1}$ and $\beta / 2$ be positive integer, the measure associated with (A.1) maps to the measure proportional to

$$
\prod_{l=1}^{N} \xi_{l}^{\tilde{\lambda}_{1}}\left(1-\xi_{l}\right)^{\tilde{\lambda}_{2}} \prod_{1 \leq j<k \leq N}\left(\xi_{k}-\xi_{j}\right)^{\beta} d \xi_{1} \cdots d \xi_{N}
$$

with

$$
\tilde{\lambda}_{1}=-b_{1}-1-(\beta / 2)(N-1), \quad \tilde{\lambda}_{2}=b_{1}+i b_{2} .
$$

In particular, this tells us that the averages

$$
\left\langle\prod_{l=1}^{N}\left(x-e^{i \theta_{l}}\right)^{\alpha} s_{\mathcal{K}}\left(e^{i \theta_{1}}, \ldots, e^{i \theta_{N}}\right)\right\rangle
$$

with respect to (A.1) satisfy the same recurrences (2.2) as the corresponding averages (2.1) for the Jacobi ensemble. We remark that this same conclusion can be reached by direct application of integration by parts as used in [17], [19, §4.6], without the need to assume $b_{1}$ and $\beta / 2$ are positive integers.

We would like to make use of the recurrences satisfied by (A.4) to provide a recursive computational scheme for the circular ensemble gap probability

$$
E_{N}^{C}(0 ;(0, \phi) ; \beta)=\lim _{\mu \rightarrow 0} \frac{1}{\mathcal{N}_{\beta, N}} \int_{\phi}^{2 \pi} d \theta_{1} \cdots \int_{\phi}^{2 \pi} d \theta_{N} \prod_{l=1}^{N} e^{i \theta_{l} \mu} \prod_{1 \leq j<k \leq N}\left|e^{i \theta_{k}}-e^{i \theta_{j}}\right|^{\beta},
$$

where the parameter $\mu$ is introduced for later convenience. As for the Jacobi gap probability $E_{N}^{\mathrm{J}}\left(0 ;(s, 1) ; \lambda_{1}, \lambda_{2}, \beta\right)$ in the parameter range (3), the case of $\beta$ a positive integer is special in this regard. Thus with the ordering

$$
R_{N}: 2 \pi>\theta_{1}>\theta_{2}>\cdots>\theta_{N}>\phi,
$$

analogous to (1.14) we have

$$
\prod_{1 \leq j<k \leq N}\left|e^{i \theta_{k}}-e^{i \theta_{j}}\right| \beta=\chi \prod_{l=1}^{N} e^{-i \theta_{l} \beta(N-1) / 2} \prod_{1 \leq j<k \leq N}\left(e^{i \theta_{j}}-e^{i \theta_{k}}\right)^{\beta},
$$


where here $\chi$ is a phase, $|\chi|=1$, which has a polynomial structure. In particular for $\beta$ a positive integer, the multidimensional integral is a finite series in powers of $e^{i \phi}$, although taking the limit $\mu \rightarrow 0$ will introduce factors which are polynomials in $\phi$ itself.

From the working of the above paragraph, it suffices to specify a computational scheme for the integrals

$$
\int_{R_{N}} d \theta_{1} \cdots d \theta_{N} \prod_{l=1}^{N} e^{i \theta_{l} \tilde{\mu}} \prod_{1 \leq j<k \leq N}\left(e^{i \theta_{j}}-e^{i \theta_{k}}\right)^{\beta}
$$

This is done as for the computation of $E_{N}^{\mathrm{J}}\left(0 ;(s, 1) ; \lambda_{1}, \lambda_{2}, \beta\right)$ in the parameter range (3), as detailed in the discussion about 2.25). Actually it is a little simpler, since instead of making repeated use of 1.16 , we only require use of

$$
\int_{0}^{\phi} d \theta e^{i \theta v}=\frac{1}{i v}\left(e^{i \phi v}-1\right)
$$

This one dimensional integral is required for the initial condition $N=1$, and then the evaluation of the case $N=n$ of A.8 from knowledge of the explicit fractional power series form of

$$
\int_{R_{n-1}} d \theta_{1} \cdots d \theta_{n-1} \prod_{l=1}^{n-1} e^{i \theta_{l} \tilde{\mu}}\left(e^{i \theta_{l}}-e^{i \phi}\right)^{\beta} \prod_{1 \leq j<k \leq n-1}\left(e^{i \theta_{j}}-e^{i \theta_{k}}\right)^{\beta} .
$$

This in turn is deduced from knowledge of the same expansion for the case $N=n-1$ of A.8), then applying the recurrence 2.2 with parameters compatible with (A.3).

For $\beta$ even it is possible to establish a direct relation between $E_{N}^{J}\left(0 ;(s, 1) ; \lambda_{1}, \lambda_{2}, \beta\right)$ and $E_{N}^{\mathrm{C}}(0 ;(0, \phi) ; \beta)$. First, from the definitions we have

$$
\begin{aligned}
E_{N}^{\mathrm{J}}\left(0 ;(s, 1) ; 0, \lambda_{2}, \beta\right)=E_{N}^{\mathrm{J}}\left(0 ;(0,1-s) ; 0, \lambda_{2}, \beta\right) & \\
& =\frac{1}{J_{N, \lambda_{2}, 0, \beta}} \int_{1-s}^{1} d x_{1} \cdots \int_{1-s}^{1} d x_{N} \prod_{l=1}^{N} x_{l}^{\lambda_{2}} \prod_{1 \leq j<k \leq N}\left|x_{k}-x_{j}\right|^{\beta} .
\end{aligned}
$$

For $\beta$ even, the expansion (1.14) is valid without any need to order the variables. Substituting in the above gives the formula

$$
E_{N}^{\mathrm{J}}\left(0 ;(s, 1) ; 0, \lambda_{2}, \beta\right)=\frac{1}{J_{N, \lambda_{2}, 0, \beta}} \sum_{\kappa} \alpha_{\kappa} \prod_{l=1}^{N} \frac{1}{\kappa_{l}+\lambda_{2}+1}\left(1-(1-s)^{\kappa_{l}+\lambda_{2}+1}\right) .
$$


Consider now $E_{N}^{C}$. From the definitions,

$$
\begin{aligned}
& E_{N}^{C}(0 ;(0, \phi) ; \beta)=E_{N}^{C}(0 ;(2 \pi-\phi, 2 \pi) ; \beta) \\
& =\frac{1}{\mathcal{N}_{\beta, N}} \int_{0}^{2 \pi-\phi} d \theta_{1} \cdots \int_{0}^{2 \pi-\phi} d \theta_{N} \prod_{1 \leq j<k \leq N}\left|e^{i \theta_{k}}-e^{i \theta_{j}}\right| \beta .
\end{aligned}
$$

For $\beta$ even we can use the expansion (A.7) without the need to impose the ordering (A.6). This shows

(A.11)

$$
E_{N}^{\mathrm{C}}(0 ;(0, \phi) ; \beta)=\frac{\tilde{\chi}}{\mathcal{N}_{\beta, N}} \lim _{\mu \rightarrow 0} \sum_{\kappa} \alpha_{\kappa} \prod_{l=1}^{N} \frac{1}{\left(\mu-(N-1) \beta / 2+\kappa_{l}\right.}\left(1-e^{-i \phi\left(\mu-(N-1) \beta / 2+\kappa_{l}\right.}\right),
$$

where $\tilde{\chi}$ has modulus 1. Comparison of (A.10) and (A.11) shows that for $\beta$ even we can map from $E_{N}^{J}$ to $E_{N}^{C}$ by setting $\lambda_{2}+1=\mu-(N-1) \beta / 2$ and $1-s=e^{-i \phi}$, then taking the limit $\mu \rightarrow 0$ and adjusting the normalisation.

\section{REFERENCES}

[1] G. Akemann, T. Guhr, M. Kieburg, R. Wegner and T. Wirtz, Completing the picture for the smallest eigenvalue of real Wishart matrices, Phys. Rev. Lett. 113 (2014), 250201

[2] T.W. Anderson, An introduction to multivariate statistics, Wiley, New York, 1958.

[3] K. Aomoto, Jacobi polynomials associated with Selberg's integral, SIAM J. Math. Analysis 18 (1987), 545-549.

[4] K. Aomoto and M. Kita, Theory of hypergeometric functions, Springer, Tokyo, Japan (2011).

[5] R. Askey, Orthogonal Polynomials and Special Functions, in: Regional Conference Series in Applied Math., 21, SIAM, 1975 .

[6] _ Some basic hypergeometric extensions of integrals of Selberg and Andrews, SIAM J. Math. Anal. 11 (1980), 938-951.

[7] _ Letter to the SIAM minisymposium "Problems and solutions in special functions",in: OP-SF NET $5 \cdot 5$ (Web resource), 1998

[8] T.H. Baker and P.J. Forrester, The Calogero-Sutherland model and generalized classical polynomials, Commun. Math. Phys. 188 (1997), 175-216.

[9] F. Bornemann, On the numerical evaluation of distributions in random matrix theory: a review, Markov Processes Relat. Fields 16 (2010), 803-866.

[10] M. Chiani, Distribution of the largest root of a matrix for Roy's test in multivariate analysis of variance, J. Mult. Analysis 143 (2016), 467-471.

[11] B. Collins, Product of random projections, Jacobi ensembles and universality problems arising from free probability, Prob. Theory Rel. Fields 133 (2005), 315-344.

[12] A.W. Davis, On the marginal distributions of the latent roots of the multivariable beta matrix, Ann. Math. Statist. 43 (1972), 1664-1669.

[13] P. Diaconis and P.J. Forrester, Hurwitz and the origin of random matrix theory in mathematics, Random Matrix Th. Appl. 6 (2017), 1730001.

[14] I. Dumitriu, Smallest eigenvalue distribution of two classes of $\beta$-Jacobi ensembles, J. Math. Phys. 53 (2012), 103301. 
[15] A. Edelman, The distribution and moments of the smallest eigenvalue of a random matrix of Wishart type, Lin. Alg. Appl. 159 (1991), 55-80.

[16] A. Edelman and B.D. Sutton, The beta-Jacobi matrix model, the CS decomposition, and generalized singular value problems, Found. Comput. Math. 8 (2008), 259-285.

[17] P.J. Forrester, Recurrence equations for the computation of correlations in the $1 / r^{2}$ quantum many body system, J. Stat. Phys. 72 (1993), 39-50.

[18] _ Quantum conductance problems and the Jacobi ensemble, J. Phys. A 39 (2006), 6861-6870.

[19] __Log-gases and random matrices, Princeton University Press, Princeton, NJ, 2010.

[20] __ Large deviation eigenvalue density for the soft edge Laguerre and Jacobi $\beta$-ensembles, J. Phys. A: Math. Theor. 45 (2012), 145201.

[21] P.J. Forrester and T.D. Hughes, Complex Wishart matrices and conductance in mesoscopic systems: exact results, J. Math. Phys. 35 (1994), 6736-6747.

[22] P.J. Forrester and M. Ito, Difference system for Selberg correlation integrals, J. Phys. A: Math. Theor. 43 (2010), 175202.

[23] P.J. Forrester and S. Kumar, Recursion scheme for the largest $\beta$-Wishart-Laguerre eigenvalue and Landauer conductance in quantum transport, J. Phys. A: Math. Theor. 52 (2019), 42LTo2.

[24] P. J. Forrester and A. K. Trinh, Optimal soft edge scaling variables for the Gaussian and Laguerre even ensembles, Nucl. Phys. B 938 (2019), 621-639.

[25] _ Finite size corrections at the hard edge for the Laguerre $\beta$ ensemble, Stud. Appl. Math. 143 (2019), $315-336$.

[26] P.J. Forrester and E. M. Rains, A Fuchsian matrix differential equation for Selberg correlation integrals, Commun. Math. Phys. 309 (2012), 771.

[27] P.J. Forrester and S.O. Warnaar, The importance of the Selberg integral, Bull. Am. Math. Soc. 45 (2008), 489-534.

[28] D. Holcomb and G.R.M. Flores, Edge Scaling of the $\beta$-Jacobi ensemble, J. Stat. Phys. 149 (2012), 1136-1160.

[29] A.T. James, Special functions of matrix and single argument in statistics, in Theory and Applications of Special Functions (R. A. Askey, Ed.), Academic, New York, 1975, pp. 497-520

[30] I.M. Johnstone, Multivariate Analysis and Jacobi Ensembles: Largest Eigenvalue, Tracy-Widom limits and rates of convergence, Ann. Stat. 36 (2008), 2638-2716.

[31] I.M. Johnstone and B. Nadler, Roy's largest root test under rank-one alternatives, Biometrika 104 (2017), 181-193.

[32] J. Kaneko, Selberg integrals and hypergeometric functions associated with Jack polynomials, SIAM J. Math Anal. 24 (1993), 1086-1110.

[33] S. Kumar, Recursion for the Smallest Eigenvalue Density of beta-Wishart-Laguerre Ensemble, J. Stat. Phys. 175, (2019) 126.

[34] S.N. Majumdar A. Pal and G. Schehr, Extreme eigenvalue statistics of correlated random variables: a pedagogical review, Physics Reports 840 (2020), 1-32.

[35] S.N. Majumdar and G. Schehr, Top eigenvalue of a random matrix: large deviations and third order phase transition, J.Stat. Mech. 2014 (2014) Po1012.

[36] M.L. Mehta, Random matrices and the statistical theory of energy levels, Academic Press, New York, 1967.

[37] _ Problem 74-6, Three multiple integrals, SIAM Rev. 16 (1974), 256-257

[38] M.L. Mehta and F.J. Dyson, Statistical theory of the energy levels of complex systems. V, J. Math. Phys. 4 (1963), 713-719. 
[39] L. Moreno-Pozas, D. Morales-Jimenez and M.R. McKay, Extreme eigenvalue distributions of Jacobi ensembles: new exact representations, asymptotics and finite size corrections, Nucl. Phys. B 947 (2019), 114724.

[40] W.G. Morris, Constant Term Identities for Finite and Affine Root Systems: Conjectures and Theorems, Ph.D. thesis, Univ. Wisconsin-Madison, 1982.

[41] R.J. Muirhead, Aspects of multivariate statistical theory, Wiley, New York, 1982.

[42] T. Nagao and P.J. Forrester, The smallest eigenvalue distribution at the spectrum edge of random matrices, Nucl. Phys. B 509 (1998), 561-598.

[43] D. Ostrovsky, A Review of conjectured laws of total mass of Bacry-Muzy GMC measures on the interval and circle and their applications, Rev. Math. Phys. 30 (2018), 1830003.

[44] I. Rumanov, Painlevé representation of Tracy-Widom $\beta$ distribution for $\beta=6$, Commun. Math. Phys. 342 (2016), 843-868.

[45] A. Selberg, Bemerkninger om et multipelt integral, Norsk. Mat. Tidsskr. 24 (1944), 71-78.

[46] C.A. Tracy and H. Widom, Fredholm determinants, differential equations and matrix models, Commun. Math. Phys. 163 (1994), 33-72.

[47] Wolfram Research Inc. Mathematica Version 12 (2019).

School of Mathematics and Statistics, ARC Centre of Excellence for Mathematical \& Statistical Frontiers, University of Melbourne, Victoria 3010, Australia

E-mail address: pjforr@unimelb.edu.au

Department of Physics, Shiv Nadar University, Gautam Buddha Nagar, Uttar Pradesh 201314, India E-mail address: skumar.physics@gmail.com 